www.jmscr.igmpublication.org

Index Copernicus Value: 79.54

ISSN (e)-2347-176x ISSN (p) 2455-0450

crossrefDOI: https://dx.doi.org/10.18535/jmscr/v7i1.141

\title{
Added Benefit of Doppler Sonography over Mammography and Ultrasonography in the Evaluation of Solid Breast Masses
}

\author{
Authors \\ Daisy Joseph ${ }^{*}$, Gayathri $\mathbf{A R}^{2}$ \\ ${ }^{1}$ Assistant Professor, Department of Radiodiagnosis, Govt Medical College, Kottayam \\ ${ }^{2}$ Senior Resident, Department of Radiodiagnosis, Govt Medical College, Kottayam \\ *Corresponding Author \\ Daisy Joseph \\ Email-dsphilipc@gmail.com
}

\begin{abstract}
Background: Breast Cancer is the most common female Cancer worldwide. Imaging of breast plays a crucial role in early detection of carcinoma breast. This study was performed to assess the nature of a solid breast lesion with mammography and gray scale ultrasonography, determine the Color Doppler characteristics of the lesion as well as correlate mammographic, gray scale and Color Doppler Sonographic findings with histopathological reports.

Materials and Methods: A Cross-sectional study was conducted in 50 patients with palpable breast lesionswho underwent mammography, gray scale ultrasonography and Color Doppler in the Department of Radiodiagnosis, Government Medical College, Thrissur. Their data were collected and statistically analyzed.

Results: By combined mammography and sonographic evaluation it was seen that 25 cases had benign morphology and 25 had malignant morphology. 7 out of 25 cases with benign morphology proved to be malignant on histology. Blood Flow was demonstrated in most of the malignant lesions giving a sensitivity of $93.7 \%$, specificity of $66.6 \%$ and positive predictive value of $83.3 \%$ for determination of malignancy. Number of vessels, high impedance and turbulent flow were found to have predictive value in differentiating benign from malignant lesions.

Conclusion: Being a screening procedure for breast malignancy, mammography alone will not give definitive diagnosis in patients with breast lump. If it is combined with high frequency ultrasound, colour \& power Doppler study, accuracy in predicting malignancy increases. Doppler by itself is of little use in evaluation of solid breast lesions. When used in conjunction with conventional examinations it can provide a more accurate characterization of certain lesions. The detection of vessels inside a lesion is significantly linked with neovascularization of malignancy.

Keywords: Breast cancer; mammography; Color Doppler; Neovascularization.
\end{abstract}

\section{Introduction}

Breast Cancer is the most common female Cancer worldwide with an estimated 1.38 million new cases diagnosed in 2008 representing $23 \%$ of all cancer in women. It is estimated that by 2030 the global burden of breast cancer will increase to over 2 million new cases per year and in India it is $200000 /$ year $^{(1)}$.

The incidence of Breast cancer is rising in India and is now the second most common cancer 
diagnosed in women after cervical cancer. It is estimated that in 2008 there were 115251 new cases of breast cancer with an age standardized incidence rate of 22.9 per $100000^{(2)}$.

Imaging of breast plays a crucial role in early detection of carcinoma breast. Imaging starts from screening mammogram to most modern technique of Elastography and MRI. Conventional film screen mammography is a widely accepted diagnostic technique to evaluate clinically suspected breast lesions and also for screening for breast cancer. Risk of radiation is a major drawback of mammography.

As the specificity of mammography is low, imaging modalities such as high frequency ultrasound, colour and power Doppler, contrast enhanced colour, Doppler and finally Elastogrphy were also introduced. Currently MRI is an adjunct to mammography and ultrasonography especially in local staging of breast cancer \& for differential diagnosis of atypical cases.

The major role of ultrasound in breast evaluation is to distinguish between cystic and solid masses and to further characterize solid masses into benign or malignant categories. In addition ultrasound is the primary modality for guided biopsies and all interventional procedures in breast disease. A high frequency, high resolution, linear transducers with dynamic focussing capabilities is recommended for ultrasound examination. However ultrasound reigns supreme as the primary modality which is not only rapid and radiation free, but is also cost effective for screening and diagnosis and image guided intervention in breast diseases.

Color Doppler evaluation for the presence and pattern of vascularity further helps characterization of breast masses in to benign and malignant varieties. Tumor angiogenesis and gradient of vascularity of the mass remains the basic of color Doppler. The vascularity is important in elucidating tumor growth because a tumor cannot grow more than 1-2 mm without recruitment of new capillary blood vessels.
Biopsy and histopathological examination still remain the gold standard for confirmation of malignancy.

\section{Materials and Methods}

A cross sectional study was done in, Dept. of Radiodiagnosis, Medical College Thrissur,during the period September 2010 to November 2011. All patients with a palpable breast lesion undergoing mammography were included in the study. Only patients with sonographically detectable lesions were included in further Doppler studies. Patients who didn't give consent and those with recurrences/distant metastasis were excluded from study.

\section{Study Instrument}

Mammogram were taken using LORAD, A HOLOGIC COMPANY, M-IV and sonographic examinations were done with PHILIPS, EN VISOR HD, VERSION C .High frequency linearprobe was used for the sonomammography

\section{Study Procedure}

After obtaining clearance from Ethical committee and Institutional Research Board, study was commenced. After noting the history and clinical examination, the patients underwent mammography.

Mediolateral oblique (MLO) and Craniocaudal (CC) views were taken in all cases. Criteria For Adequate Positioning In MLO View was defined as the visulaisation of pectoralis major muscle down to the level of nipple, Inclusion of axillary tail and inframammary tissue. The $\mathrm{CC}$ view was considered adequate when anteroposterior distance on both sides was symmetric.

\section{Benign Criteria Studied with Mammography}

1) Low density

2) Smooth margins

3) Coarse calcifications

4) Larger than clinical size

The patients were subjected to sonographic and doppler examination following mammography. The lesions were classified into benign and malignant, for each modality. Doppler 
examination were carried out in sonographically detected lesions. Standardized machine settings were selected to give maximum sensitivity for detection of small Doppler shifts (highest transmit power, persistence, longest velocity scale, lowest filter setting). Number of vessels were counted and each was evaluated by Doppler after adequate angle correction. If intratumoral vascularity was detected the number of vessels were counted zero to five or greater. Study of vessels with pulsed Doppler sonography was performed to obtain spectral wave forms for up to five different vessels. The resistive index (RI), pulsatality index (PI) and S/D ratio were calculated The highest RI, PI and S/D ratio obtained were considered the RI and PI and S/D ratio values for the lesions.

\section{Doppler Wave Forms were Categorized In to Four Types}
1) Low impedance pulsatile
2) High impedance pulsatile
3) Turbulent
4) Venous

\section{Low Impedance Pulsatile Signals}

Low level of pulsatility and relatively high levels of diastolic flow as is seen in arteries that supply organs with low peripheral resistance.

\section{High Impedance Pulsatile Signals}

High impedance pulsatile signals have high pulsatility with low frequency diastolic components or no flow in diastole.

\section{Turbulent Signals}

Turbulent signals were those that showed broadening of the Doppler spectrum as might be seen in a stenotic Vessels, indicating a wide range of frequencies.

\section{Venous Signals}

Venous flow was defined as continuous flow throughout the cardiac cycle with minimal or no variation in velocity.

\section{Outcome Measures}

At the end of the study each patient would have the following information.

Morphology of the tumor
Categorization into benign/malignant by USG \& mammography

Doppler study - Blood flow indices, type of vessels.

Histo-pathological confirmation of nature.

The patients can be broadly classified into two categories: 1. Those with benign lesions and

2. Those with malignant lesion. The difference in Doppler characteristics of each of these groups are identified.

\section{Data collection and analysis}

Details of each patient was collected in a proforma and entered in MS Excel. All the data coded and entered in MS Excel, and analyzed using SPSS 16. Tests used were proportions, chisquare tests means and independent $t$ test for difference in means.

\section{Results}

By combined mammography and sonographic evaluation, it was seen that 25 cases had benign morphology and 25 had malignant morphology. 7 out of 25 cases with benign morphology proved to be malignant on histology.

Age was considered as a dichotomous variable. 6 out of 8 cases under 40 years of age showed benign lesions, 2 turned out to be malignant. 42 patients were above 40 years of age. 30 out of them had malignant breast lesions while only 12 were benign.

Blood Flow was demonstrated in 6 of 18 benign lesions and 30 of 32 malignant lesions giving a sensitivity of $93.7 \%$, specificity of $66.6 \%$ and positive predictive value of $83.3 \%$ for determination of malignancy based on the presence of any blood flow.

In a significantly greater proportion of malignant lesions, three or more vessels were counted. $60 \%$ of malignant lesions which showed blood flow had more than three vessels within the lesion compared with $33.3 \%$ of benign lesion.

Of the lesions that showed flow, most common spectrum was of a low impedance pulsatile pattern. This was seen in $86.6 \%$ of malignant 
lesion and $100 \%$ of benign lesions which showed blood flow. Hence, was not discriminatory.

However turbulent high impedance pulsatile and venous signals were all significantly more likely to be seen in malignant lesions.

None of the benign lesions showed pulsatile or turbulent signals while $50 \%$ of those benign cases with vascularity showed venous flow.

Among malignant lesions 26\% displayed high impedance pulsatile, $16 \%$ turbulent and $53.3 \%$ showed venous flow.

$\chi 2$ analysis showed that malignant lesions were more likely to display more signal types of flow. More over presence of high impedance and turbulent flow was seen only in malignant lesions.

\section{False Negative Findings}

7 malignant lesions showed benign morphology in mammography and ultrasound examination. All of them were found to be vascular. Of these 4 were infiltrating duct carcinoma grade II, 2 were infiltrating duct carcinoma grade III and 1 was proliferative breast disease with atypical epithelial cells.

2 malignant lesions showed no evidence of blood flow on colour Doppler analysis and showed malignant morphology in USG and mammographic study, these 2 were infiltrative ductal carcinoma on histology

\section{False Positive}

None of the sonologically malignant lesions demonstrated benign morphology.

Of the benign lesions which showed vascularity most of them had only one vessel which showed low impedance flow. Four of these lesions were fibroadenomas and two were inflammatory in origin.

A logistic regression was computed to determine if the presence of flow allowed differentiation of benign from malignant lesions. A second analysis focused only on lesions with blood flow and tried to identify the blood flow parameters that were most useful in differentiating benign from malignant lesions. Each variable was assessed separately.

Number of vessels, high impedance and turbulent flow were found to be of predictive value in differentiating benign from malignant lesions.

RI Values of benign lesions with vascularity was in the range 0.5- to 0.69 and that of malignant lesions of 0.6 to 0.89 which was statistically significant.

The PI values of malignant lesions were also found to be higher being 1.32 to 1.56 when compared to benign 0.6-1.2.

Similarly S/D ratio of benign were 2-3.5 while those of malignant were in the range of 3.55.3.The findings showed that higher RI, PI, and $\mathrm{S} / \mathrm{D}$ value were more in favour of malignancy.

\begin{tabular}{|l|c|c|c|}
\hline & \multicolumn{2}{|c|}{ Histopathological Diagnosis } & Pvalue \\
\hline Variable & Benign(N=18) & Malignant(N=32) & \\
\hline $\begin{array}{l}\text { Age (years) } \\
<40\end{array}$ & $6(33.3 \%)$ & $2(6.25 \%)$ & 0.019 \\
$>40$ & $12(66.6 \%)$ & $30(93.75 \%)$ & \\
\hline $\begin{array}{l}\text { Mammographic \& Sonographic } \\
\text { Morphology }\end{array}$ & & & \\
Benign & $18(100 \%)$ & $7(21.8 \%)$ & 0.000 \\
Malignant & $0(0 \%)$ & $25(78.1 \%)$ & \\
\hline Bloodflow & $6(33.3 \%)$ & $30(93.75 \%)$ & 0.000 \\
\hline No. of vessels & & & \\
$<3$ & $4(66.6 \%)$ & $12(40 \%)$ & 0.230 \\
$>3$ & $2(33.3 \%)$ & $18(60 \%)$ & \\
\hline Signal Type & & & \\
\hline Low Impedence & $6(100 \%)$ & $26(86.6 \%)$ & 0.002 \\
\hline High Impedence & $0(0 \%)$ & $8(26.6 \%)$ & 0.021 \\
\hline Turbulent & $0(0 \%)$ & $5(16.6 \%)$ & \\
\hline Venous & $3(50 \%)$ & $16(53.3 \%)$ & 0.019 \\
\hline Resistive Index & $0.5-0.69$ & $0.6-0.89$ & 0.000 \\
\hline Pulsatality Index & $0.6-1.2$ & $1.32-1.56$ & 0.000 \\
\hline S/D ratio & $2-3.5$ & $3.5-5.3$ & 0.000 \\
\hline
\end{tabular}




\section{JMSCR Vol||07||Issue||01||Page 808-815||January}

Fig-1 : Malignant Lesion with Multiple Vessels

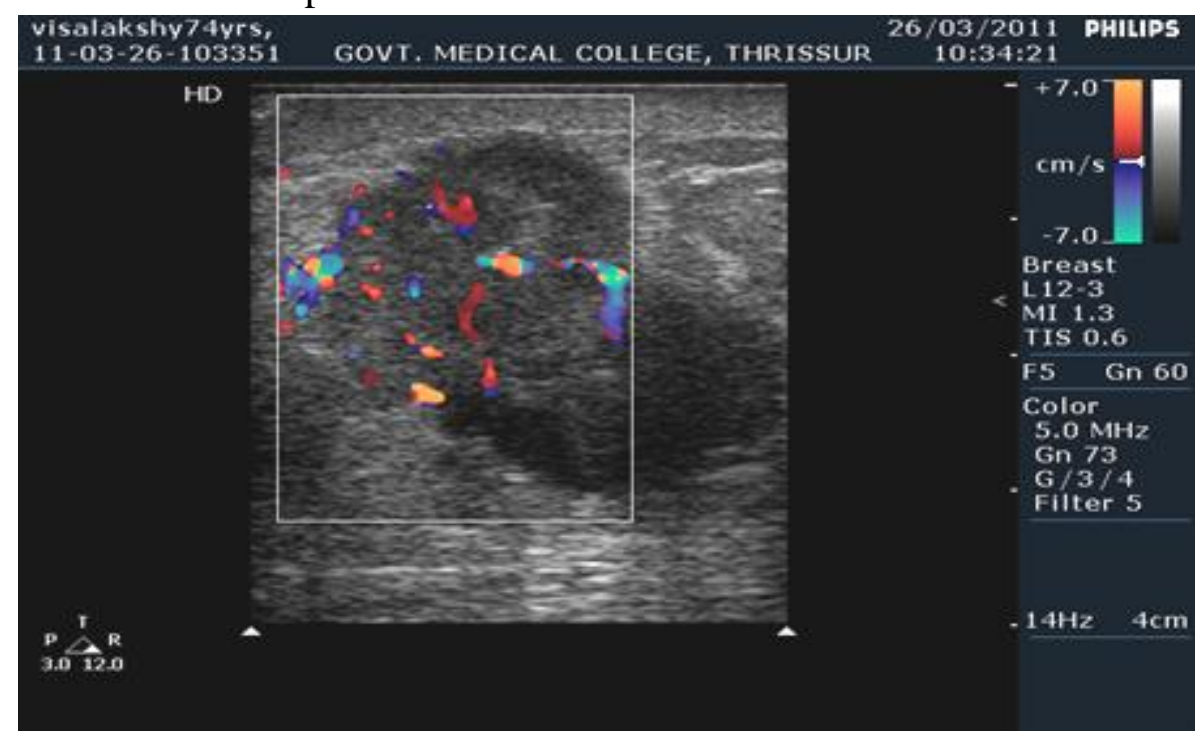

Fig: 2: Malignant Lesion with Spectral Wave form Showing a Low Impedance Vessel

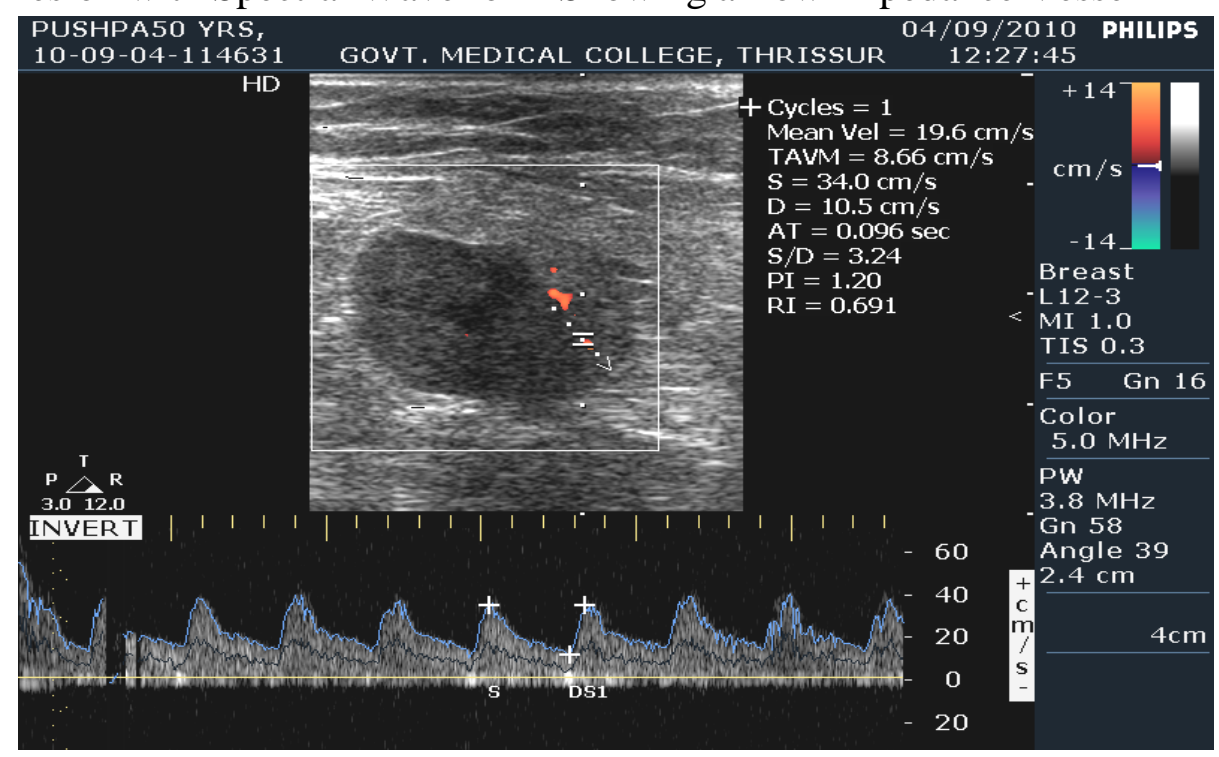

Fig: 3: Malignant Lesion with Spectral Wave Form Showing a High Impedance

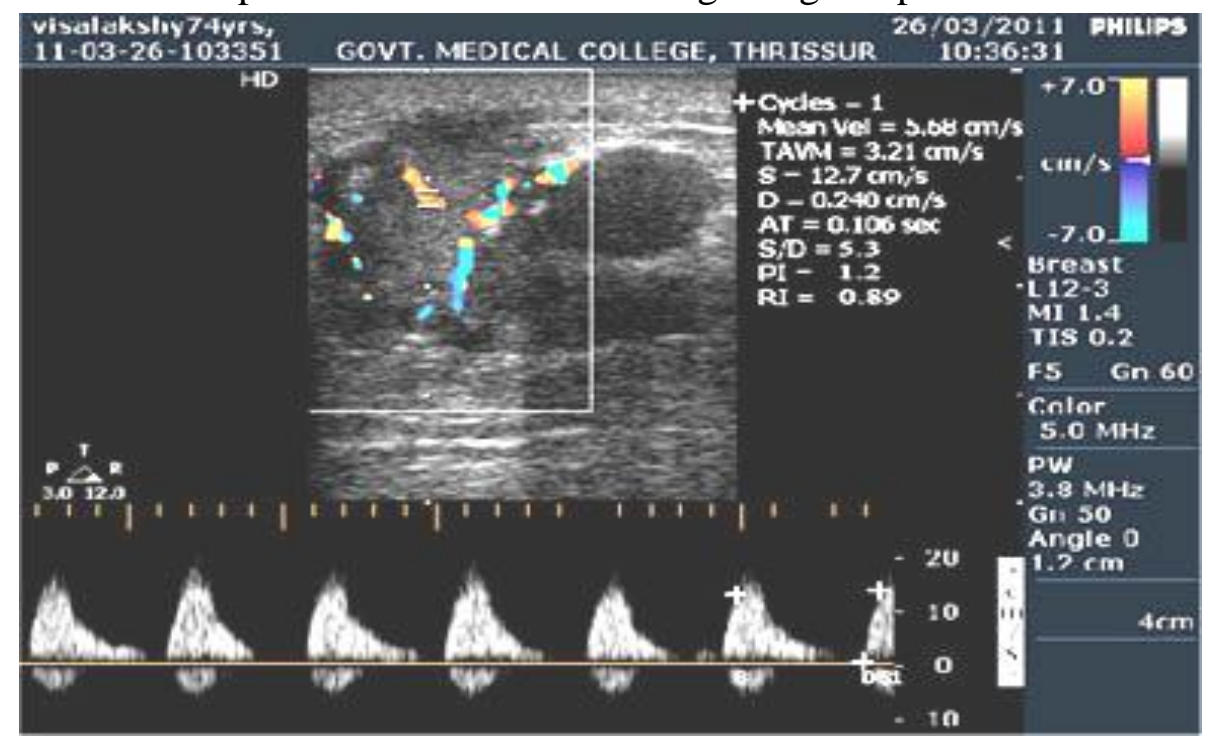




\section{JMSCR Vol||07||Issue||01||Page 808-815||January}

Fig: 4 Malignant Lesion Showing Venous Flow

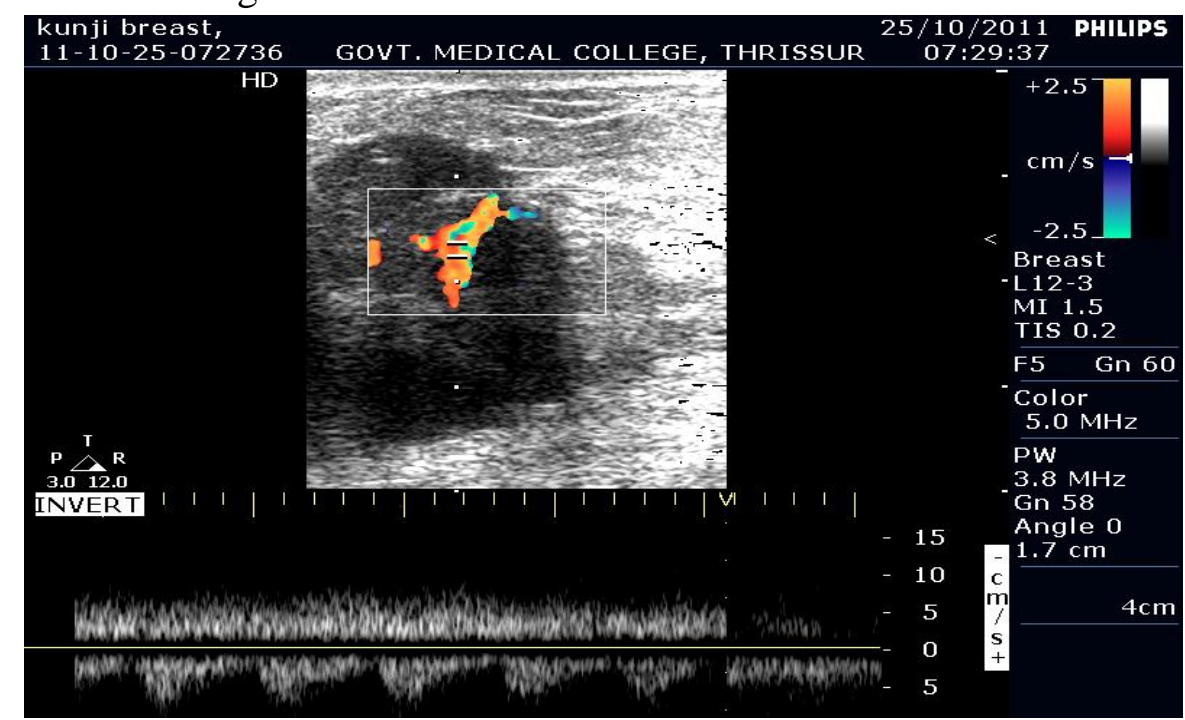

Fig: 5 Malignant Lesion Showing a vessel with Turbulent Flow

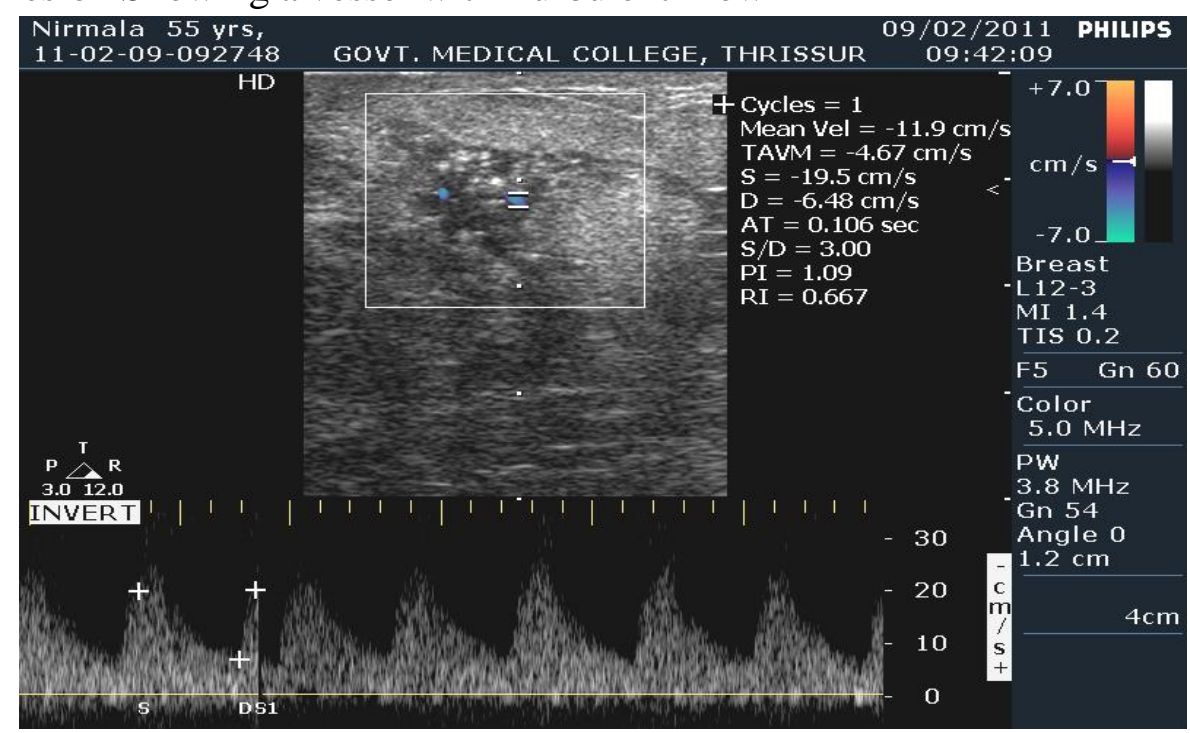

Fig: 6 Malignant Lesion with no Vessels Detected

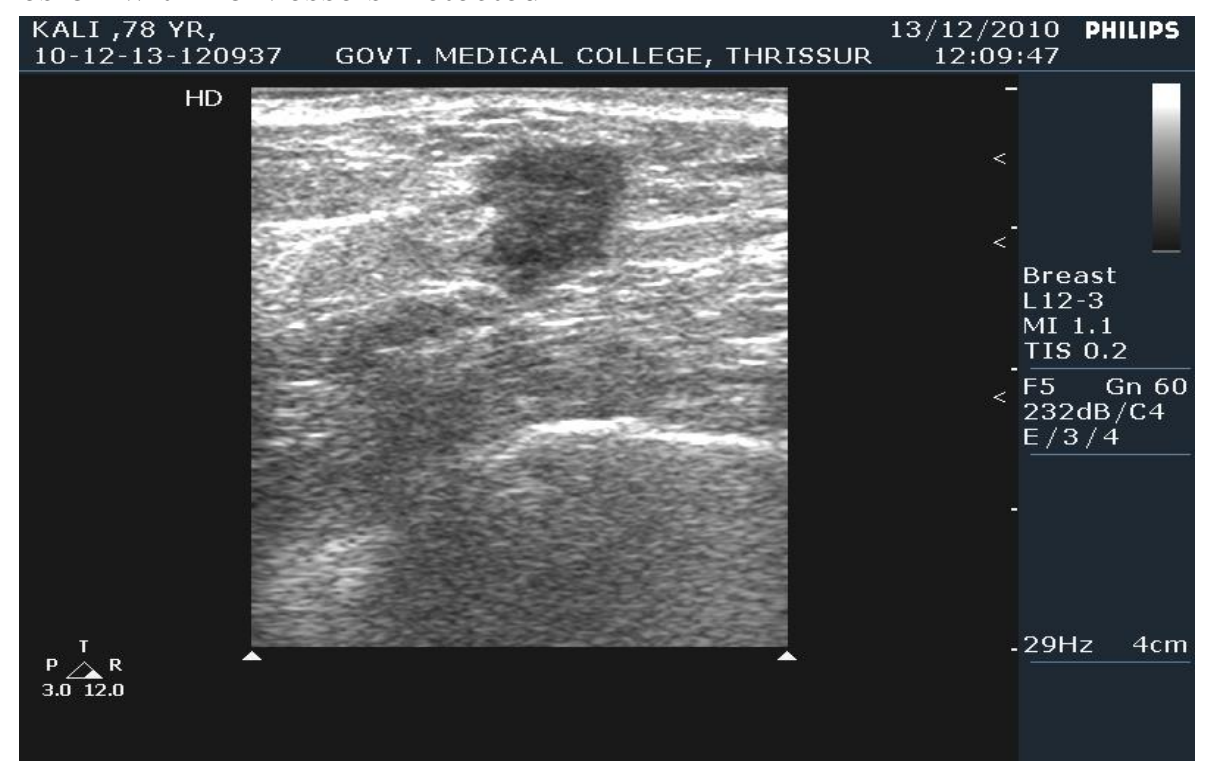




\section{JMSCR Vol||07||Issue||01||Page 808-815||January}

Fig: 7 Benign Lesions with No Flow

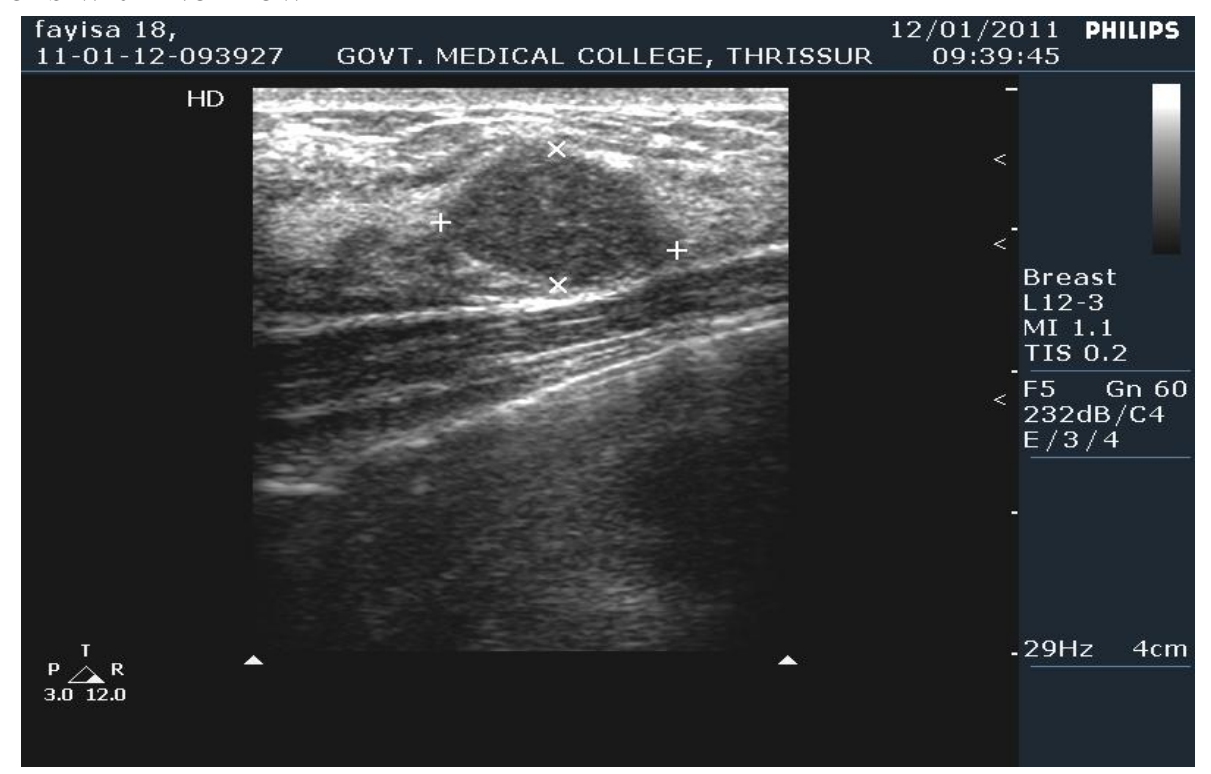

Fig: 8 Benign Lesions with a Low Impedance Vessel

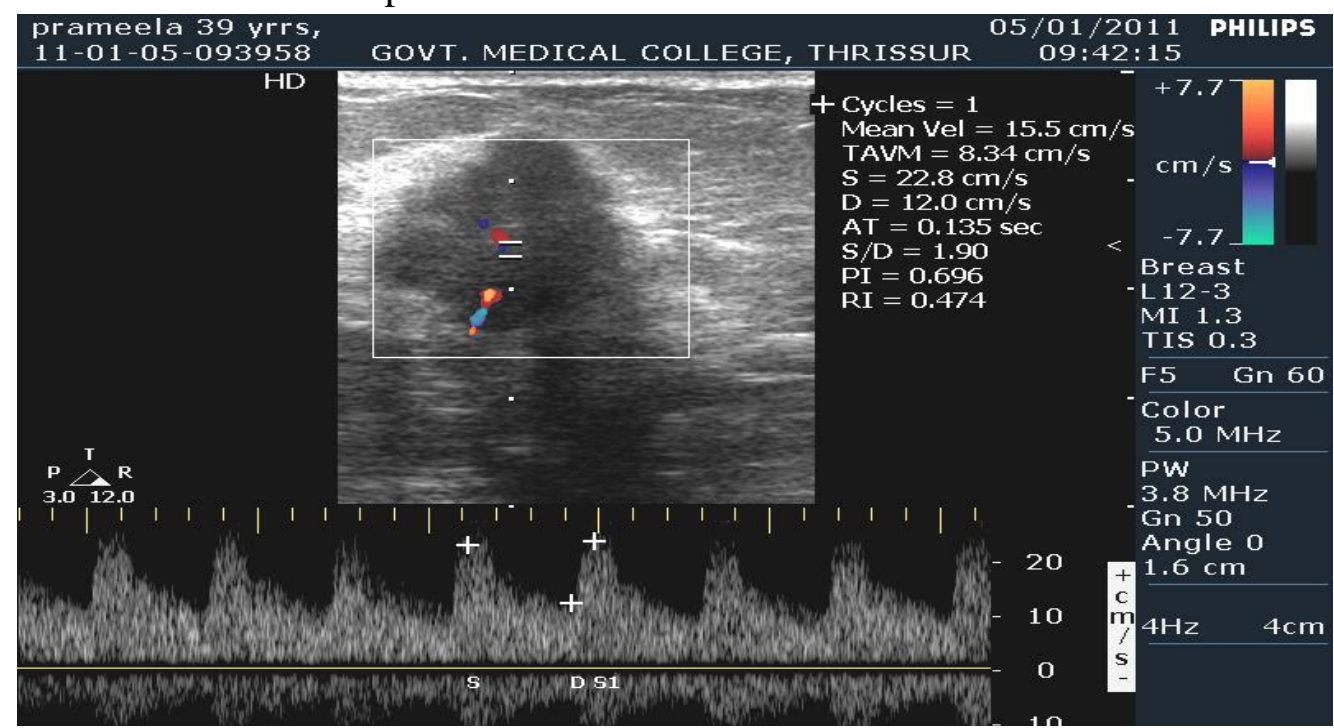

\section{Discussion}

Tumour angiogenesis plays an important role in the growth and extension of malignant neoplasms including those of the breast. Until now various parameters of Doppler sonography has been analyzed to determine their usefulness in the diagnoses of breast lesions. The detection of vascularity in the lesion was the first Doppler feature used to differentiate between benign and malignant tumors and has shown a significant association with malignancy. The logistic regression analysis in our data confirms that the malignant nature of the tumor itself is related to higher detection rates of vessels. Previous reports have shown a marked variability for detecting vessels in a tumor as a sign of malignancy with sensitivities ranging from $73 \%$ to $98 \%$, specificities from $16 \%$ to $90 \%$, positive predictive values from $41 \%$ to $85 \%$ and negative predictive values from $47 \%$ to $99 \% .{ }^{(3)}$. Variability may be due to differences in proportions of the various lesions.

In this study it was able to detect vessels in $93 \%$ of breast cancers whereas only $33 \%$ of benign lesions showed vascularity. Higher percentage of detection of vascularity in malignant lesions when compared to other studies may be because only palpable lesions were included here. In those lesions in which flow velocity curves could be obtained there was significant differences between 
the flow patterns for malignant and benign lesions, the former having much higher RI, PI, and $\mathrm{S} / \mathrm{D}$ values than latter. This is correlating well with the study conducted by Jose L. Del Cura et $\mathrm{al}^{(4)}$.

The increase in resistance is related to the existence of occlusions and stenosis in the tumor vessel network structure produced by vascular encasement due to tumor growth. These values showed significant differences between benign and vascular lesions. Most of the studies done earlier showed similar higher values in malignant lesions but most of them showed overlapping with benign lesions rendering it little useful as a tool for differentiation.

For lesions that showed blood flow, consideration of pattern of flow was a helpful indicator of malignancy as the presence of high impedance and turbulent vessels and to some extent the presence of venous signals were seen in malignant lesions. This was correlatiing with the studies done by M.M.J. McNicholas et $\mathrm{al}^{(3)}$

All the malignant lesions which showed benign morphology in USG and mammographic study were detected to be vascular while those malignant lesions which were a vascular showed distinct malignant morphology in USG and mammogram.

\section{Conclusion}

Being a screening procedure for breast malignancy, mammography alone will not give definitive diagnosis in patients presenting with breast lump. If it is combined with high frequency ultrasound, colour \& power Doppler study, it shows high accuracy in predicting malignancy.
In conclusion Doppler by itself is of little use when it comes to evaluating solid breast lesions. When used in conjunction with conventional examinations it can provide a more accurate characterization of certain lesions. The detection of vessels inside a lesion is significantly linked with neovascularisation of malignancy. The presence of turbulent and high impedance flow on spectral imaging is a sign although infrequent, is of high positive predictive value. Finally Histopathological conclusion is the gold standard for differentiation of tissues.

\section{Bibliography}

1. Ferlay J, Shin H-R, Bray F, Forman D, Mathers C, Parkin DM. Estimates of worldwide burden of cancer in 2008: GLOBOCAN 2008. Int. J. Cancer. 2010 Dec 15;127(12):2893-917.

2. GLOBOCAN 2008: Cancer Incidence and Mortality Worldwide [Internet]. [cited 2018 Jun 5]. Available from: https://www.iarc.fr/en/mediacentre/iarcnews/2010/globocan2008.php

3. M. M. J. McNicholas, P. M. Mercer, J. C. Miller. Color Doppler Sonography in the Evaluation of Palpable Breast Masses [Internet]. [cited 2018 Jun 5]. Available from: https://www.ajronline.org/doi/pdf/10.2214 /ajr.161.4.8372754

4. Del Cura JL, Elizagaray E, Zabala R, Legórburu A, Grande D. The Use of Unenhanced Doppler Sonography in the Evaluation of Solid Breast Lesions. American Journal of Roentgenology. 2005 Jun 1;184(6):1788-94. 